Article

\title{
The Effects of Thermoplastic Polyurethane on the Structure and Mechanical Properties of Modified Polypropylene Blends
}

\author{
Ting An Lin ${ }^{1}$, Ching-Wen Lou ${ }^{2,3,4,5}$ and Jia-Horng Lin $4,5,6,7,8,9, *$ \\ 1 Laboratory of Fiber Application and Manufacturing, Department of Fiber and Composite Materials, \\ Feng Chia University, Taichung 40724, Taiwan; annlin9247@gmail.com \\ 2 School of Textiles, Tianjin Polytechnic University, Tianjin 300387, China; cwlou@ctust.edu.tw \\ 3 Graduate Institute of Biotechnology and Biomedical Engineering, Central Taiwan University of Science \\ and Technology, Taichung 40601, Taiwan \\ 4 Department of Chemistry and Chemical Engineering, Minjiang University, Fuzhou 350108, China \\ 5 College of Textile and Clothing, Qingdao University, Qingdao 266071, China \\ 6 Department of Fiber and Composite Materials, Feng Chia University, Taichung 40724, Taiwan \\ 7 Innovation Platform of Intelligent and Energy-Saving Textiles, School of Textiles, Tianjin Polytechnic University, \\ Tianjin 300387, China \\ 8 School of Chinese Medicine, China Medical University, Taichung 40402, Taiwan \\ 9 Department of Fashion Design, Asia University, Taichung 41354, Taiwan \\ * Correspondence: jhlin@fcu.edu.tw; Tel.: +886-4-2451-0871
}

Received: 25 October 2017; Accepted: 29 November 2017; Published: 3 December 2017

\begin{abstract}
In this study, a melt-compounding process was used to produce ordinary polypropylene (PP)/thermoplastic polyurethane (TPU) blends and modified impact-resistant polypropylene (MPP)/TPU blends. The influences of TPU on the blending morphology, melting behavior, crystallization behavior, and mechanical properties of these blends were investigated. Differential scanning calorimetry results show that using TPU influences the crystalline and melting behavior of PP/TPU blends, but not of MPP/TPU blends. X-ray diffraction analysis indicated that the presence of TPU neither influences the crystalline structure of PP nor creates new crystalline peaks. The mechanical property test results revealed that PP/TPU blends demonstrate better tensile strength, Young's modulus, and flexural strength, whereas MPP/TPU blends exhibit higher energy absorption and impact strength. This study is expected to produce optimal products with the preferred continuous and dispersive phases for end users, designing multiple and compatible functionalities of blends.
\end{abstract}

Keywords: polymer composites; polypropylene; modified polypropylene; thermoplastic polyurethane; impact strength; melt-blending method

\section{Introduction}

Polypropylene (PP) is a commonly used plastic material composed of semi-crystalline polymer and exhibits easy processing, chemical resistance, high rigidity, light weight, and a reasonable, relatively low cost $[1,2]$. However, PP has some disadvantages, such as a large shrinkage ratio, great brittleness at low temperature, and low impact resistance, and thus its development is limited. There are two effective methods of improving the low impact resistance. One method is to modify the PP copolymer by adding ethylene polypropylene rubber (EPR) or multiple-side-chained ethylene groups to enhance the impact resistance of PP-based blends. The other method is to blend elastomer with PP, obtaining synergistic impact strength [3].

The blending of polymers has gained much attention in both industrial and academic fields. A single specific polymer, or more than one polymer, can be added to polymer blends in order to 
acquire certain properties. Thermoplastic polyurethane (TPU) is a polymer composed of both a hard segment (HS) and a soft segment (SS). An HS consists of urethane groups, while a SS consists of a polyol that includes ester groups or ether groups. TPU is featured as having an elasticity of rubber as well as high mechanical properties of plastic materials [4-6]. Therefore, using TPU effectively improves the mechanical properties and impact strength [7].

Bajsic et al. indicated that TPU/PP blends with $80 \mathrm{wt} \%$ TPU and $20 \mathrm{wt} \%$ PP resulted in poor mechanical properties, and the tensile strength, elongation at break, and Young's modulus were 17.4 MPa, 7.0\%, and $645.3 \mathrm{MPa}$, respectively. These results can be attributed to the immiscibility and incompatibility between PP and TPU, which led to an inhomogeneous phase with poor interaction [8]. $\mathrm{Qu}$ et al. examined the mechanical properties of TPU/PP blends with various contents of TPU (10-90 $\mathrm{wt} \%)$ and found that a U-shaped mechanical property curve formed when TPU content increased. The test results indicated that the tensile strength of blends was significantly dependent on the blending ratios. Moreover, due to a weak phase interaction and the disruption of TPU interchange hydrogen bonding, a minimum tensile strength was revealed with respect to blends with $60 \mathrm{wt} \% \mathrm{TPU}$ and thus led to the propagation of cracks at the weak phase. In addition, the impact strength was obviously improved by a higher content of TPU, indicating that stress could be effectively transferred by a strong interface [9]. Therefore, the blending ratio of TPU and PP played a key role in the mechanical properties of the blends, such as tensile strength, elongation at break, Young's modulus, and impact strength.

In this study, PP and modified impact-resistant polypropylene (MPP), the former of which is a homopolymer and the latter of which is an impact copolymer, were used as the polymer matrix. MPP was expected to reduce the amount of TPU since TPU is an expensive polymer material. In most references, the blending ratios were set at 80:20, 70:30, and 50:50 for PP and TPU, and the mechanical properties of the PP/TPU blends were further discussed. Our results indicated that the tensile properties of PP/TPU blends decreased as more TPU was added. Some problems resulted from the larger intrinsic structure of TPU and from interphase interaction and transformations [8], so the proportion of TPU was set at $10 \mathrm{wt} \%$. In the meantime, the pure PP and pure MPP were used as control groups, and the effects of using TPU on the blending morphology, melting behavior, crystalline behavior, and mechanical properties of polymer blends were investigated.

\section{Experimental}

\subsection{Materials}

Polypropylene (PP, YUNGSOX 1080, Formosa Plastics Corporation, Taipei, Taiwan) is a homopolymer that has a density of $0.90 \mathrm{~g} / \mathrm{cm}^{3}$ and a melt index of $10 \mathrm{~g} / 10 \mathrm{~min}$ (ISO1133). Modified impact-resistant polypropylene (MPP, J-750HP, Prime Polymer Co., Ltd., Tokyo, Japan) is a copolymer that has a density of $0.91 \mathrm{~g} / \mathrm{cm}^{3}$ and a melt index of $14 \mathrm{~g} / 10 \mathrm{~min}$ (ISO1133). Thermoplastic polyurethane (TPU, HE-3285ALE, Headway Polyurethane Co., Ltd., Hsinchu, Taiwan) is an ester-type copolymer that has a density of $1.23 \mathrm{~g} / \mathrm{cm}^{3}$ and a melt index of $7.03 \mathrm{~g} / 10 \mathrm{~min}\left(175^{\circ} \mathrm{C}, 2.16 \mathrm{~kg}\right)$.

\subsection{Preparation}

$\mathrm{PP} / \mathrm{TPU}$ and MPP/TPU blends were made using a melt-compounding process. The blending ratios for both PP/TPU and MPP/TPU blends were 90:10 (90 wt \% of PP or MPP and $10 \mathrm{wt} \%$ of TPU). A single-screw extruder (SEVC-45, Re-Plast Extrder Corp., Miaoli, Taiwan) was used for melt-compounding. The temperature parameters of the barrels were $200,210,210$, and $210{ }^{\circ} \mathrm{C}$, and the rotary speed was $25 \mathrm{rpm}$. The schematic diagram of the manufacturing process is shown in Figure 1. The PP/TPU polymer melts were drawn and cooled in a water bath to make them uniform and evenly mixed. The resulting samples were PP90/TPU10 and MPP90/TPU10 blends. The blends were processed using an injection mold in order to form different blends. PP100 and pure MPP100 
were used as control groups. Tensile tests, an impact test, and a flexural strength test were conducted to examine the mechanical properties of the resultant products.

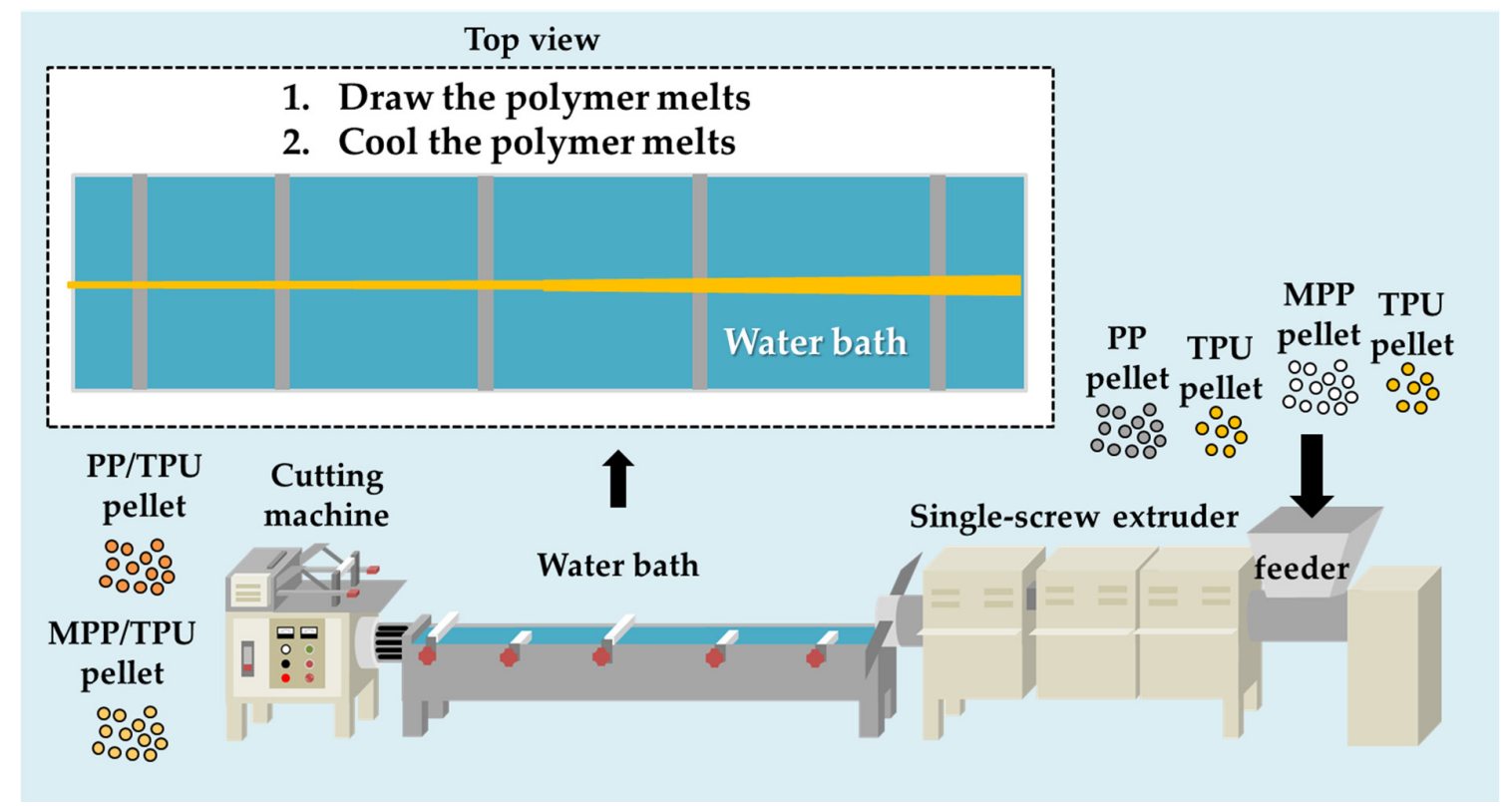

Figure 1. Schematic diagram of the manufacturing process. PP: polypropylene; MPP: modified impact-resistant polypropylene; TPU: thermoplastic polyurethane.

\subsection{Tests}

\subsubsection{Scanning Electron Microscopy (SEM)}

SEM analysis (Phenom Pure+, Phenom world, Jing Teng Tech Limited Company, New Taipei City, Taiwan) were used to observe the fractured samples collected from the impact strength test. Moreover, the distribution of TPU in PP90/TPU10 blends and MPP90/TPU10 blends were also observed using SEM.

\subsubsection{Differential Scanning Calorimetry (DSC)}

A differential scanning calorimeter (Q200, TA Instruments, New Castle, DE, USA) was used to evaluate the influence of TPU on the crystallization temperature, crystallization enthalpy, melting temperature, and melting enthalpy of MPP90/TPU10 and PP90/TPU10.

\subsubsection{X-ray Diffraction (XRD)}

An X-ray diffractometer (MXP3, Mac Science, Yokohama, Japan) was used to measure the crystalline structure of the samples. The diffraction angle was between 10 and $30^{\circ}$, and the test rate was $2^{\circ} / \mathrm{min}$.

\subsubsection{Mechanical Properties}

Tensile tests were conducted as specified by ASTM D638, while the flexural strength test was conducted as specified by ASTM D790-10. An Instron 5566 (Instron, Canton, MA, USA) was used for the tensile and flexural tests. A notched Izod impact strength test was performed to measure the impact energy and impact strength of samples using an Izod impact strength tester (CPI, ATLAS, Mount Prospect, Chicago, IL, USA) as specified by ASTM D256-10. For each test, 10 samples for each specification were tested in order to determine the mean in a given condition. 


\section{Results and Discussion}

\subsection{Morphology of PP/TPU and MPP/TPU Blends}

Figure 2A shows that PP100 had a smooth fractured surface, and the growth path of cracks was short. The majority of cracks were parallel and classified as typical fragility [10]. PP is a polymer composed of mono propylene units, and its molecular chains are regularly arranged. Therefore, PP exhibits lower impact resistance due to its regular arrangement of polymer chains. MPP is a copolymer with many side chains in its molecular structure, leading to a better impact resistance. Figure 2B reveals that MPP100 had cracks on the fractured surface and that the cracks were aligned in different directions. Moreover, the fractured surface of MPP100 possessed a multilayered structure and rough surface, indicating that MPP100 can effectively absorb externally exerted impact energy [10]. Figure 2C illustrates that, for PP90/TPU10, TPU particles at a dispersive phase were evenly distributed in PP at a continuous phase. When an impact force was exerted at PP90/TPU10, the stress was concentrated on TPU, and the blend started to deform and absorbed impact energy. Figure 2D shows that, when MPP90/TPU10 is impacted, $10 \mathrm{wt} \%$ TPU can form tremendous micro-cracks or shear bands that are able to dissipate impact energy, thereby strengthening the toughness of MPP90/TPU10.

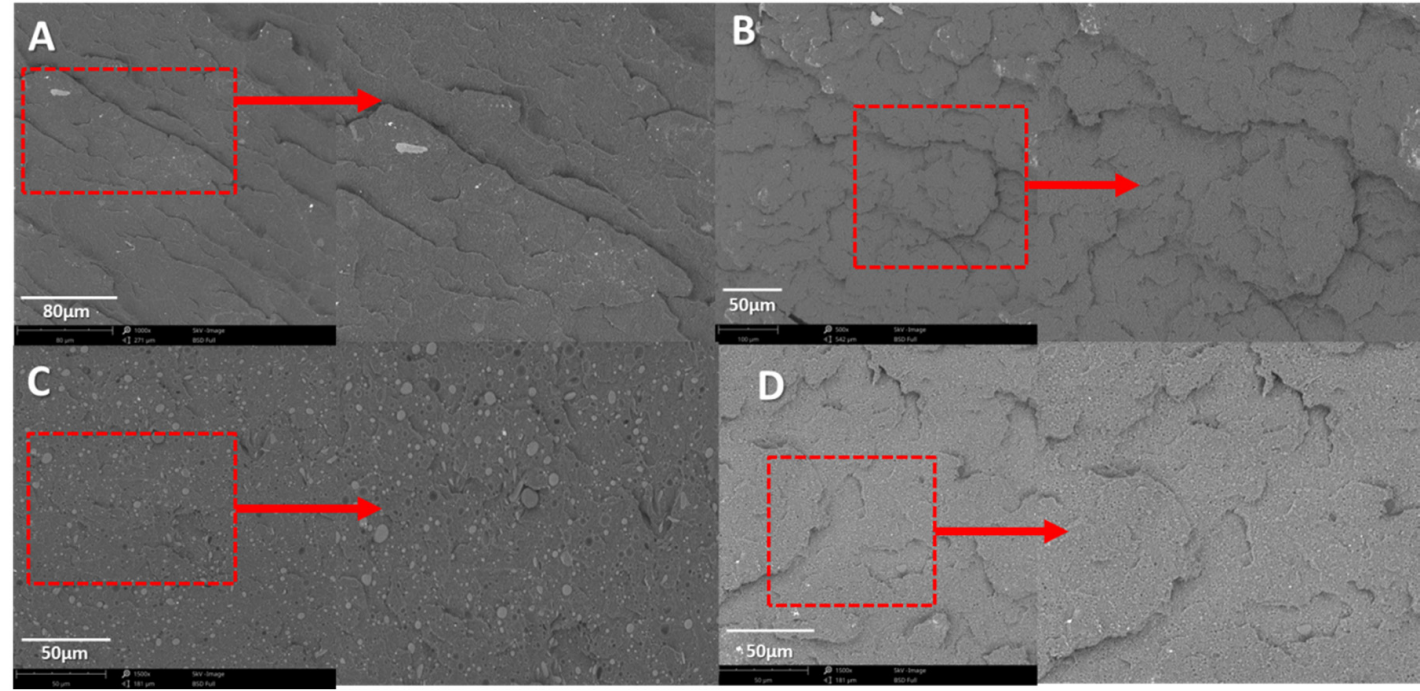

Figure 2. Fractured surface of (A) PP100 $(1000 \times)$, (B) MPP100 $(500 \times),($ C) PP90/TPU10 $(1500 \times)$, and (D) MPP90/TPU10 $(1500 \times)$.

\subsection{Melting and Crystalline Behaviors}

PP is a semi-crystal polymer, whose melting and crystallization properties depend on its structure, the copolymer, and the fillers. The melting and crystalline behaviors of all blends are indicated in Table 1 and Figure 3. Based on the DSC results, TPU exhibited a crystallization temperature of $62.41^{\circ} \mathrm{C}$, a crystallization enthalpy of $5.82 \mathrm{~J} / \mathrm{g}$, a melting point of $145.69{ }^{\circ} \mathrm{C}$, and a melting enthalpy of $8.49 \mathrm{~J} / \mathrm{g}$, and all of these values, compared with other groups, are much lower. Figure 3A shows that PP100 and MPP100 possess similar melting points, which were $165.29{ }^{\circ} \mathrm{C}$ and $165.64{ }^{\circ} \mathrm{C}$, respectively. Moreover, the melting point did not fluctuate after the addition of $10 \mathrm{wt} \% \mathrm{TPU}$, indicating that TPU did not affect the crystalline structure or stability of PP. In contrast, $10 \mathrm{wt} \%$ TPU decreaseed the melting enthalpy of PP90/TPU10 by $15 \mathrm{~J} / \mathrm{g}$. This is ascribed to the presence of TPU that was an amorphous polymer. Compared with PP100, PP90/TPU required relatively little energy to hamper the reaction between molecules, contributing to a greater mobility of the PP molecular chain. Figure 3B reveals no significant differences in the crystallization temperature or the crystallization enthalpy between MPP100 and MPP90/TPU10. MPP is a modified PP polymer with low crystallization, whereas TPU is an amorphous 
polymer. Their (MPP90/TPU10) combination caused a slight decrease in the crystallization temperature and crystallization enthalpy of MPP90/TPU10. In addition, the combination of PP and amorphous polymer TPU in PP90/TPU10 resulted in an increasing crystallization temperature. Compared to PP100, the crystallization of PP90/TPU10 took longer. PP has a polymorphism that can be divided into three crystalline structures: an $\alpha$ crystal form of the monoclinic system, a $\beta$ crystal form of the hexagonal system, and a $\gamma$ crystal form of the orthorhombic system [11]. Under common processing conditions, isotactic PP often shows an $\alpha$ crystalline form. Figure 3 C shows that, in the XRD test, when $2 \theta$ was $14.1^{\circ}, 16.8^{\circ}, 18.5^{\circ}, 21.3^{\circ}$, and $21.8^{\circ}$, the corresponding Bragg diffraction characteristic peaks of PP were accompanied by crystal plane diffraction of (110), (040), (130), (111), and (131) [1,12-14]. The results illustrated that, for PP90/TPU10 and MPP/TPU, the diffraction peak remained the same, indicating that adding TPU did not create a new crystalline structure $[15,16]$. Moreover, both PP/TPU at (110) and MPP90/TPU10 at (040) had higher strengths. There were no significant differences in other peak values, suggesting that TPU did not function as a nucleating agent in the PP or MPP matrix and thus did not cause any significant difference in the crystalline phases of PP or MPP [11].

Table 1. Melting and crystalline behavior of PP/TPU blends and MPP/TPU blends. PP: polypropylene; MPP: modified impact-resistant polypropylene; TPU: thermoplastic polyurethane.

\begin{tabular}{ccccc}
\hline Sample Codes & $\begin{array}{c}\text { Crystallization } \\
\text { Temperature }\left({ }^{\circ} \mathbf{C}\right)\end{array}$ & $\begin{array}{c}\text { Crystallization } \\
\text { Enthalpy }(\mathbf{J} / \mathbf{g})\end{array}$ & $\begin{array}{c}\text { Melting } \\
\text { Temperature }\left({ }^{\circ} \mathbf{C}\right)\end{array}$ & $\begin{array}{c}\text { Melting } \\
\text { Enthalpy (J/g) }\end{array}$ \\
\hline TPU100 & 62.41 & 5.82 & 145.69 & 8.49 \\
PP100 & 115.24 & 106.20 & 165.29 & 115.40 \\
PP90/TPU10 & 121.62 & 96.23 & 165.13 & 100.10 \\
MPP100 & 129.56 & 83.61 & 165.77 & 88.27 \\
MPP90/TPU10 & 130.20 & 83.71 & 165.64 & 87.22 \\
\hline
\end{tabular}
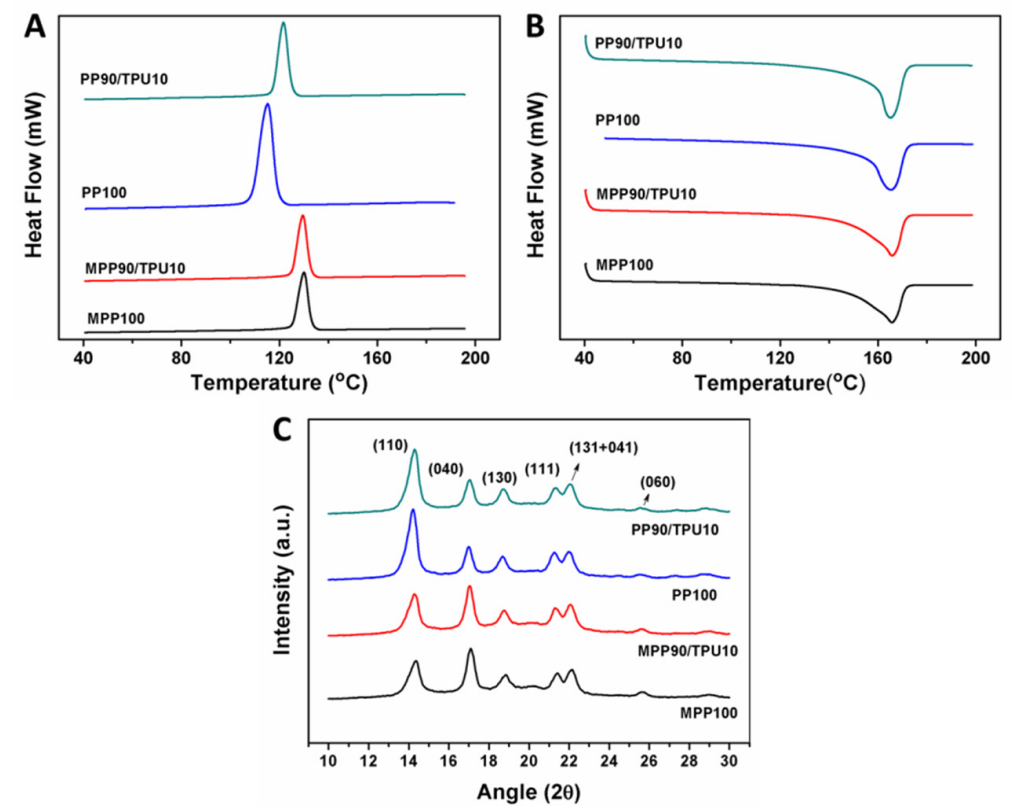

Figure 3. Melting and crystalline behavior of PP/TPU and MPP/TPU blends: (A) heating curve, (B) cooling curve, and (C) X-ray Diffraction (XRD) pattern.

\subsection{Mechanical Behaviors}

Figure $4 \mathrm{~A}, \mathrm{~B}$ show the tensile behavior and flexural strength of the experimental group and the control group. PP100 and PP90/TPU10 had areater tensile strength than MPP100 and MPP90/TPU10. PP100 was composed of highly aligned molecular chains and thus had greater crystallinity, and its 
tensile strength reached $37.12 \mathrm{MPa}$. In addition, PP90/TPU10 possessed higher elongation than PP100, which was ascribed to TPU. TPU is a thermoplastic elastomer that provides PP90/TPU10 with greater elongation. To sum up, the elongation at break could be ranked from highest to lowest as MPP90/TPU10, MPP100, and then PP90/TPU10. MPP with a great toughness and thermoplastic elastomer TPU had a synergistic effect, demonstrated by the highest elongation at break of MPP90/TPU10.
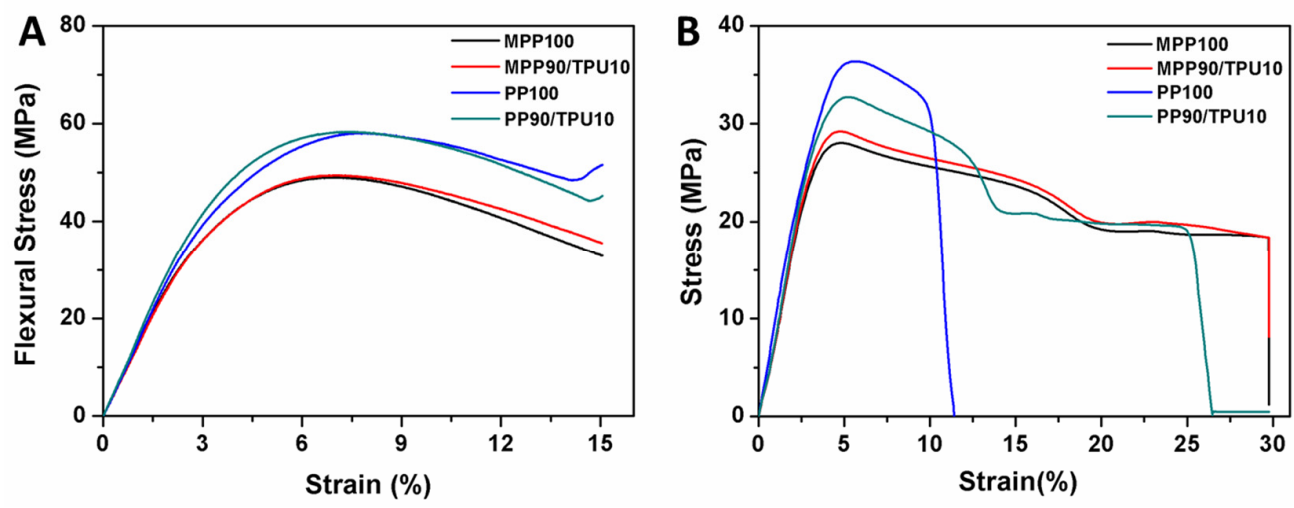

Figure 4. Tensile and flexural behavior of PP/TPU blends and MPP/TPU blends: (A) tensile properties and (B) flexural properties.

Table 2 shows that MPP100 and MPP90/TPU10 exhibited higher tensile strength, Young's modulus, and flexural strength than PP100 and PP90/TPU10. Moreover, PP100 outperformed PP90/TPU10 because PP at the continuous phase had a lower bonding level due to the presence of TPU, which compromised the mechanical properties. On the other hand, MPP100 and MPP90/TPU10 could bear more impact energy and possessed a higher impact strength than PP100 and PP90/TPU10. Due to modification, MPP exhibited a greater toughness than PP, and thus could withstand greater impact energy and showed higher impact strength. In particular, MPP90/TPU10 could absorb an impact energy of $43.3 \mathrm{~J}$, and MPP100 had an impact strength of $8.48 \mathrm{~kJ} / \mathrm{m}^{2}$. Nevertheless, compared with MPP100, MPP90/TPU10 still had a lower impact strength. This was ascribed to modified impact-resistance polypropylene (i.e., MPP) that contained more ethylene groups that was incompatible with TPU. The differences in polarity between MPP and TPU is high, and the presence of PP may break the hydrogen bond of TPU, resulting in lower mechanical properties [10].

Table 2. Mechanical properties of PP/TPU and MPP/TPU blends.

\begin{tabular}{cccccc}
\hline Sample Codes & $\begin{array}{c}\text { Tensile Strength } \\
\mathbf{( M P a )}\end{array}$ & $\begin{array}{c}\text { Young's Modulus } \\
\mathbf{( M P a )}\end{array}$ & Energy (J) & $\begin{array}{c}\text { Flexural Strength } \\
\mathbf{( M P a )}\end{array}$ & $\begin{array}{c}\text { Impact Strength } \\
\mathbf{( k J / \mathbf { m } ^ { 2 } )}\end{array}$ \\
\hline MPP100 & $29.19 \pm 1.93$ & $974 \pm 40.32$ & $32.82 \pm 8.34$ & $49.06 \pm 1.92$ & $8.48 \pm 0.55$ \\
MPP90/TPU10 & $29.79 \pm 1.55$ & $977 \pm 26.67$ & $43.33 \pm 10.24$ & $49.88 \pm 0.78$ & $4.93 \pm 0.25$ \\
PP100 & $37.12 \pm 0.55$ & $1046.79 \pm 37.47$ & $21.01 \pm 2.68$ & $58.60 \pm 1.39$ & $2.76 \pm 0.21$ \\
PP90/TPU10 & $32.36 \pm 0.86$ & $1016.80 \pm 9.89$ & $23.15 \pm 2.56$ & $57.93 \pm 0.34$ & $3.49 \pm 0.88$ \\
\hline
\end{tabular}

\section{Conclusions}

In this study, TPU was added to PP or MPP to form blends, and the blending morphology, crystallization behavior, melting behavior, and mechanical properties of blends were examined. The SEM images showed that MPP100 and MPP90/TPU10 absorbed higher imapct energy. The DSC and XRD results revealed that the addition of TPU did not affect the strucutre of PP/TPU blends but influenced its melting and crystallization enthalpy. The mechanical property test results showed that, among the blends, PP100 and PP90/TPU10 had greater tensile strength, a greater Young's modulus, and a greater flexural strength, while MPP100 and MPP90/TPU10 absorbed greater impact energy 
and had higher impact strength. It was surmised that MPP at the continuous phase may harm the hydrogen bond of TPU at the dispersive phase, which jeopardized its intrinsic high toughness. This study proposes a flexibly feasible design of polymer blends, which can be freely adjusted to suit the requirements of products and satisfy the user end.

Acknowledgments: The authors would like to thank Ministry of Science and Technology of Taiwan, for supporting this research under Contract MOST 106-2622-E-035 -013 -CC3.

Author Contributions: In this study, the concepts and designs for the experiment, all required materials, as well as processing and assessment instrument were provided by Jia-Horng Lin. Data were analyzed, and the experimental results examined, by Ching-Wen Lou. The experiment was conducted, and the text composed, by Ting An Lin.

Conflicts of Interest: The authors declare no conflict of interest.

\section{References}

1. Cho, D.; Zhou, H.J.; Cho, Y.; Audus, D.; Joo, Y.L. Structural properties and superhydrophobicity of electrospun polypropylene fibers from solution and melt. Polymer 2010, 51, 6005-6012. [CrossRef]

2. Xiao, J.M.; Chen, Y.A. New micro-structure designs of a polypropylene (pp) composite with improved impact property. Mater. Lett. 2015, 152, 210-212. [CrossRef]

3. Jeong, J.O.; Lim, Y.M.; Park, J.S. Improving thermal stability and mechanical performance of polypropylene/polyurethane blend prepared by radiation-based techniques. Eur. Polym. J. 2017, 94, 366-375. [CrossRef]

4. Hossieny, N.J.; Barzegari, M.R.; Nofar, M.; Mahmood, S.H.; Park, C.B. Crystallization of hard segment domains with the presence of butane for microcellular thermoplastic polyurethane foams. Polymer 2014, 55, 651-662. [CrossRef]

5. Bulatovic, V.O.; Mihaljevic, A.; Bajsic, E.G.; Holjevac, T.G. Morphology and thermal behavior of tpu/pp blends modified with maleic anhydride grafted sebs-g-ma block copolymer. Int. Polym. Proc. 2017, 32, 102-111. [CrossRef]

6. Bajsic, E.G.; Pustak, A.; Smit, I.; Leskovac, M. Blends of thermoplastic polyurethane and polypropylene. II. Thermal and morphological behavior. J. Appl. Polym. Sci. 2010, 117, 1378-1384.

7. Wang, J.S.; Chen, X.D.; Zhang, N.Q.; Rong, M.Z. Polyurethane/polyolefin blends: Morphology, compatibilization and mechanical properties. Polym. Polym. Compos. 2006, 14, 1-11.

8. Bajsic, E.G.; Smit, I.; Leskovac, M. Blends of thermoplastic polyurethane and polypropylene. I. Mechanical and phase behavior. J. Appl. Polym. Sci. 2007, 104, 3980-3985. [CrossRef]

9. Jia, S.K.; Qu, J.P.; Liu, W.F.; Wu, C.R.; Chen, R.Y.; Zhai, S.F.; Huang, Z. Thermoplastic polyurethane/polypropylene blends based on novel vane extruder: A study of morphology and mechanical properties. Polym. Eng. Sci. 2014, 54, 716-724. [CrossRef]

10. Luo, J.S.; Xu, B.P.; Yu, H.W.; Du, Y.X.; Feng, Y.H. Thermoplastic polyurethane/polypropylene blends in a co-rotating non-twin screws extruder. Fibers Polym. 2015, 16, 95-104. [CrossRef]

11. Zhang, S.L.; Zhang, Z.X.; Kim, J.K. Influence of regenerated pu on mechanical properties and thermal stability of pp. Mater. Lett. 2008, 62, 1532-1534. [CrossRef]

12. Favaro, M.M.; Branciforti, M.C.; Bretas, R.E.S. A X-ray study of beta-phase and molecular orientation in nucleated and non-nucleated injection molded polypropylene resins. Mater. Res. 2009, 12, 455-464.

13. Song, P.A.; Liu, L.N.; Huang, G.B.; Yu, Y.M.; Guo, Q.P. Largely enhanced thermal and mechanical properties of polymer nanocomposites via incorporating c-60@ graphene nanocarbon hybrid. Nanotechnology 2013, 24. [CrossRef] [PubMed]

14. Mazidi, M.M.; Aghjeh, M.K.R. Synergistic toughening effects of dispersed components in pp/pa6/epdm ternary blends; quantitative analysis of the fracture toughness via the essential work of fracture (ewf) methodology. RSC Adv. 2015, 5, 47183-47198. [CrossRef]

15. Bajsic, E.G.; Filipan, V.; Bulatovic, V.O.; Mandic, V. The influence of filler treatment on the mechanical properties and phase behavior of thermoplastic polyurethane/polypropylene blends. Polym. Bull. 2017, 74, 2939-2955. [CrossRef] 
16. Lan, Y.; Liu, H.; Cao, X.H.; Zhao, S.G.; Dai, K.; Yan, X.R.; Zheng, G.Q.; Liu, C.T.; Shen, C.Y.; Guo, Z.H. Electrically conductive thermoplastic polyurethane/polypropylene nanocomposites with selectively distributed graphene. Polymer 2016, 97, 11-19. [CrossRef] 